\title{
A Sky Dome Visualisation for Identification of Astronomical Orientations
}

\author{
Georg Zotti* Meister Eduard Gröller ${ }^{\dagger}$ \\ Institute of Computer Graphics and Algorithms, Vienna University of Technology
}

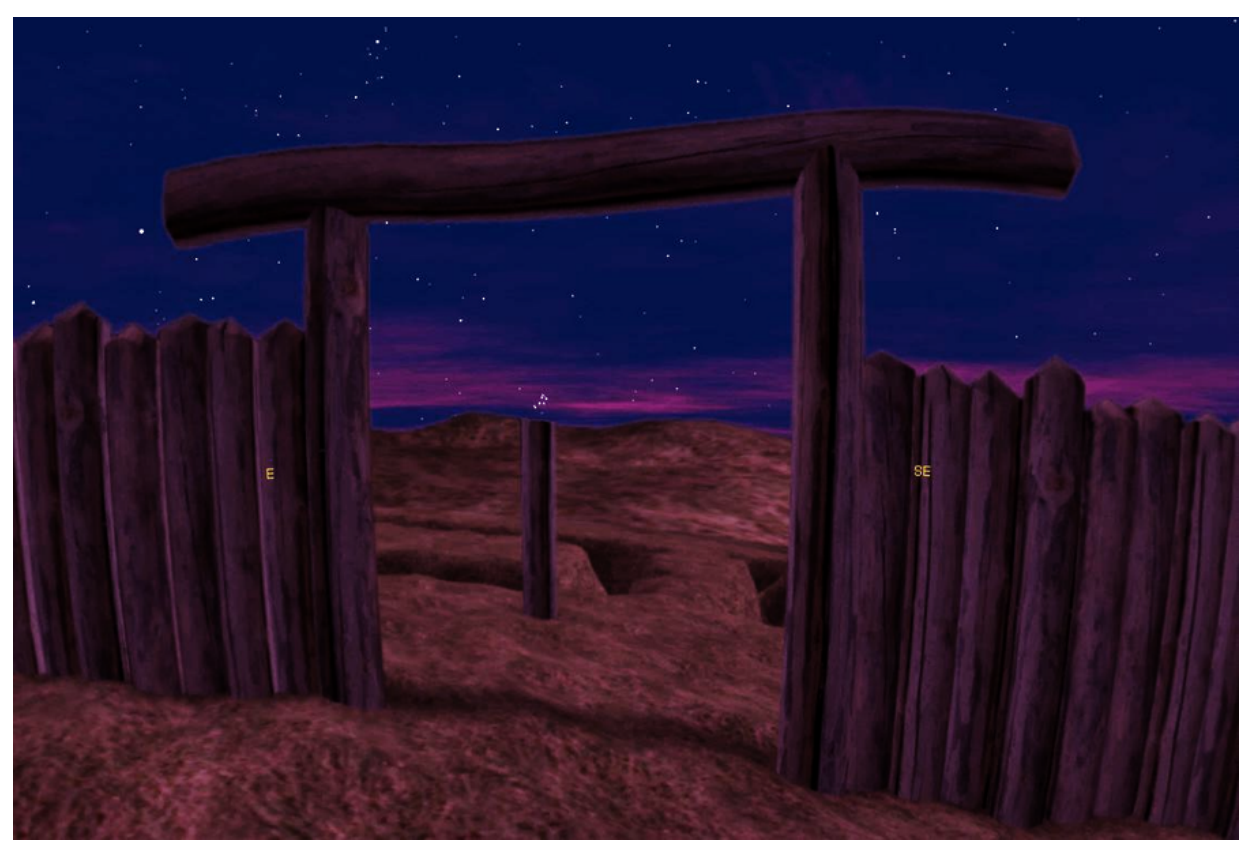

Figure 1: Virtual reconstruction of a look through one of the doors in the neolithic Kreisgrabenanlage of Steinabrunn in Lower Austria. The singular post appears to be aligned to the rising point of the Pleiades star cluster, which with their rising in the morning possibly announced the beginning of the agricultural year. Astronomical alignments like this have been identified using the method described in this paper. Screenshot from StarryNight Pro 4.5, foreground from virtual reconstruction courtesy of Imagination Computer Services, Vienna.

\section{Abstract}

It has long been known that ancient temples were frequently oriented along the cardinal directions or to certain points along the horizon where Sun or Moon rise or set on special days of the year. In the last decades, archaeologists have found evidence of even older building structures buried in the soil, with doorways that also appear to have distinct orientations.

This paper presents a novel diagram combining archaeological maps with a folded-apart, flattened view of the whole sky, showing the local horizon and the daily paths of sun, moon and brighter stars. By use of this diagram, interesting groupings of astronomical orientation directions, e.g. to certain sunrise and sunset points could be identified, which were evidently used to mark certain days of the year. Orientations to a few significant stars very likely indicated the beginning of the agricultural year in the middle neolithic period.

CR Categories: I.3.8 [Computer Graphics]: Applications; J.2 [Physical Sciences and Engineering]: Archaeology; J.2 [Physical Sciences and Engineering]: Astronomy

Keywords: Archaeology, Astronomy, data mining

\footnotetext{
*e-mail: gzotti@cg.tuwien.ac.at

†e-mail: meister@cg.tuwien.ac.at
}

\section{INTRODUCTION}

A large body of evidence shows the connection of ancient cultures to annually repeating celestial events. In many old cultures, temple axes are typically aligned either along cardinal directions (with the pyramides in Egypt as most popular kand best studied example) or towards solstice risings and settings of the sun, e.g. Persepolis [9]. Another frequently cited example is Stonehenge and many other megalithic sites, which were presumably overinterpreted by various authors, most notably Thom [10], but are largely accepted as sun- and moon-oriented temples [5]. Also medieval churches were frequently oriented towards the rising point of the Sun on the day of the church's patron [4].

Apparently the orientation of buildings has a much older tradition. Since the 1960s, aerial photography, later combined with geomagnetic prospection with highly sensitive caesium magnetometers and, where possible, successive excavations, discovered a certain class of neolithic circular enclosures: Kreisgrabenanlagen (KGA). They typically consisted of 1-3 nearly circular wooden palisade walls, surrounded by 1-3 ditches, which were $V$-shaped and up to $6 \mathrm{~m}$ deep and $12 \mathrm{~m}$ wide. The circular ditches were broken by "earth bridges", which allowed people to enter the enclosure through gaps ("doors") in the palisades. The overall diameter of these Kreisgrabenanlagen is approximately $40-180 \mathrm{~m}$. The best archaeological survey results, short of the slow and very costly direct excavation, come from measuring the minute deviations of the Earth's natural magnetic field caused by the different qualities of soil which today 


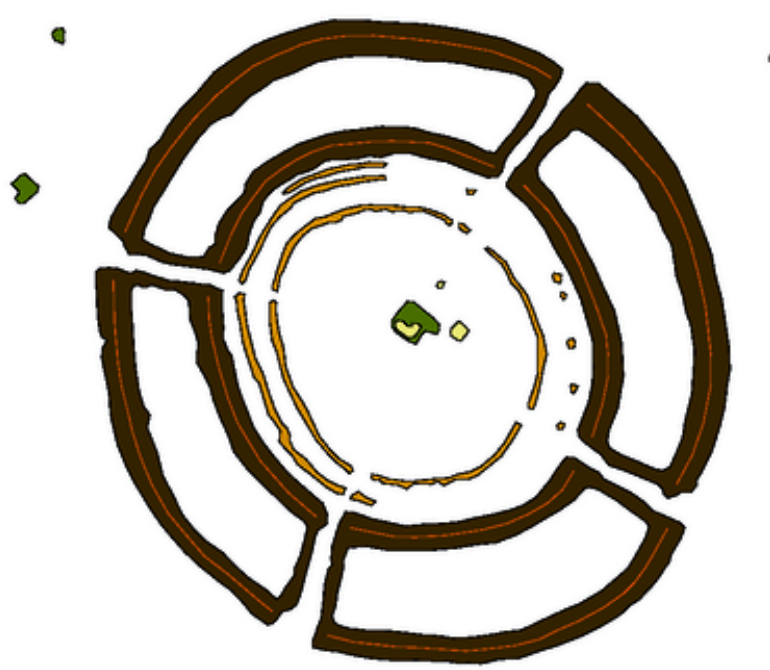

Figure 2: Archaeological map of the Kreisgrabenanlage (KGA) Steinabrunn in Lower Austria [3]. The wide brown rings indicate the circular ditches which are connected by narrower radial ditches along 4 access bridges. In the central area, traces of 3 concentric palisade walls are marked in a lighter shade of brown. Small brown dots indicate singular postholes, the green area marks a central pit. A multitude of similar KGA traces has been found in the last decades, among the many theories about their use exists the idea of astronomically oriented doorways.

fill the ditches. According to ${ }^{14} \mathrm{C}$ dating of excavated bone material, all KGAs have been erected in the very short time span between 4800 and 4500 B.C. These structures have been found over a large part of central Europe, from Hungary to northern Germany, with a concentration of over 40 such structures known in Lower Austria (e.g., Figure 2).

Since the 1980s, with increasing numbers of known KGAs, theories about their use have been discussed and rejected, spanning from cattle paddocks to fortifications. The current interpretation is their use as a place for special gatherings, cultic worship, or initiations. But, in parallel to the much younger megalithic constructions of Great Britain and Brittany [11], also some astronomical alignments have been proposed, most notably by Becker [1], who found that Kreisgrabenanlagen of Bavaria had doors and bridges aligned to rising points of the Sun at solstices.

During the preparation of the first large public exhibition on Kreisgrabenanlagen, also the theory of astronomical connections has been investigated. This paper describes what has to be taken into account for these orientation studies. Not only possible Sunrelated directions have been studied, but also Moon- and starrelated directions. A key element on the way to an interesting discovery has been a diagram combining the archaeological map with a celestial map, which allows to immediately see possible alignments.

The rest of the paper is as follows: first, important terms from astronomical phenomenology are defined, and necessary astronomical computations are pointed out. Then, a methodology is developed which shows how to apply various diagrams to questions related to astronomical orientations. Kreisgrabenanlagen are used as example, but the same idea can be applied to any building or geological structure for which an oriented map exists. Then a combined result from a multitude of KGAs is shown, which allow the postulation of an archeoastronomical theory.

\section{Celestial Positions}

To compute the position of a celestial object, we need

- the geographical position $(\lambda, \varphi)$ of the observer, where the geographical longitude $\lambda$ is positive for longitudes east of Greenwich and latitude $\varphi$ is positive on the northern hemisphere,

- the object's celestial coordinates $(\alpha, \delta)$ on the imaginary infinite celestial sphere, with the longitudinal coordinate $\alpha$ named right ascension and counted in hours instead of degrees, eastward from the First Point of Aries $\Upsilon$. This point marks the one intersection of the celestial equator with the Sun's annual path, the ecliptic, where the Sun crosses the celestial equator northward, this event defining the beginning of astronomical spring in the northern hemisphere. $\delta$ is called declination and is positive for objects north of the celestial equator.

- the time of observation. Here we have to take care not to use just zone time (maybe even further denaturalized by daylight saving rules), but local mean solar time.

From these data, we can compute an object's azimuth A, i.e., its horizontal direction, counted from north $\left(0^{\circ}\right)$ eastward (or, following a different, equally valid convention, from south westward), and $a l$ titude a. Details of the computation of positions can be found in general astronomical literature, e.g. [6].

On any day of the year, stars rise and set approximately 4 minutes earlier than on the day before. Stars surrounding the celestial pole of the observer's hemisphere are visible in every clear night and called circumpolar. Others are only visible in parts of the year, the Sun being too close to them to be observable at other times. The last time such a star is visible at dusk is called its heliacal setting. After a few weeks of invisibility due to close distance to the Sun, they emerge in the morning on the day of their heliacal rising: in the morning twilight, they appear shortly over the horizon haze, only to become too washed out a few moments later by the increasing brightness of the emerging daylight. On each successive day, they will be visible about four minutes longer. It has long been known that heliacal risings have been used by various peoples all over the world to mark specific days in the year [5], the best known example being the Egypian use of the heliacal rising of Sirius as "herald" of the Nile flood.

The rising and setting azimuths on a location's horizon depend on its geographical latitude $\varphi$, the star's declination $\delta$ and the local horizon's altitude. On the northern hemisphere, any elevation on the horizon will shift both rising and setting points southward.

In addition, for investigation of rising and setting alignments along the horizon, two phenomena caused by Earth's atmosphere have to be taken into consideration:

- refraction, causing a slight increase of altitude for objects close to the horizon. Refraction causes the Sun and Moon to appear vertically compressed when they are on the horizon, and raise both these objects by about $1 / 2$ degree on the horizon, thus causing also a slight northward shift in both rising and setting points.

This shift also applies to stars, but only the very brightest stars are visible in so low altitudes, because of the

- extinction, which reduces the objects' brightnesses, frequently making dimmer stars invisible. A star's brightness is given on a logarithmic magnitude scale, the brightest stars having slightly negative numbers. The dimmest stars observable without optical aid under best conditions are about mag 6.5 but only close to the zenith. In low altitudes, esp. close to the 


\begin{tabular}{|l|l|r|l|}
\hline Name & Constellation & mag & $a_{E}$ \\
\hline Arcturus & Bootes & -0.1 & $0^{\circ}$ \\
Vega & Lyra & 0.0 & $0^{\circ}$ \\
Capella & Auriga & 0.1 & $0^{\circ}$ \\
Procyon & Canis Minor & 0.4 & $0.3^{\circ}$ \\
Altair & Aquila & 0.8 & $0.7^{\circ}$ \\
Antares & Scorpius & 0.9 & $0.9^{\circ}$ \\
Pollux & Gemini & 1.2 & $1.2^{\circ}$ \\
Deneb & Cygnus & 1.3 & $1.3^{\circ}$ \\
\hline
\end{tabular}

Table 1: Extinction angles $a_{E}$ for a few stars[7, p.130].

horizon, the atmosphere absorbs much of the starlight. Only the brightest stars are visible on the horizon, most stars will not be visible below a certain altitude, their respective extinction angle $a_{E}$. Table 1 shows extinction angles for best conditions. Depending on humidity and dust, $a_{E}$ can be larger.

For Sun and Moon, one more aspect must be guessed: whether the alignment intended to mean the point of first resp. last visibility (i.e., upper limb), disk center or full visibility (lower limb).

\subsection{Sun}

Mirroring Earth's motion around it, the Sun annually describes a great circle on the celestial sphere, which due to Earth's tilted axis intersects the celestial equator on the First Points of Aries $\Upsilon$ and Libra $\underline{\Omega}$. The moments the sun is at these points define the beginnings of astronomical spring and autumn, respectively, in the northern hemisphere. The Sun can reach northern and southern declinations equal to the Earth's axis tilt from orbit normal, which is currently about $\varepsilon=23.5^{\circ}$, but slowly and slightly varies over millennia. Reflecting this, the annual points of sunrise and sunset span a wide arc along eastern and western horizons. Near the solstices ("standstills"), the sun rises or sets on almost the same positions for several days.

It is known from older cultures [5] that some peoples defined the seasons not beginning with solstices and equinoxes, but centering the seasons around these dates, leading to terms like "midsummer" for summer solstice. Traces of this tradition survive in today's All Saints' Day/Halloween and Candlemas as begin and end of Winter, and May Day as begin of Summer.

\subsection{Moon}

Similar to the Sun, the Moon can reach a wide area of declinations. Due to its orbit being tilted about $i=5^{\circ}$ from Earth's orbit, its extreme north and south declinations vary even wider, with outer extremes ("major standstills", $\delta=\varepsilon+i$ ) and inner extremes ("minor standstills", $\delta=\varepsilon-i$ ) [11]. Due to orbital motions, these extreme standstills occur in intervals of about 18.6 years [6].

\subsection{Stars}

Although called fixed stars, over the course of centuries and millennia, stars do change their position on the celestial sphere mostly due to

- their proper motion in space, which due to their enormous distance from the Solar system is hardly noticeable even after several thousand years, with only a handful bright exceptions, and

- precession, a motion of Earth's axis counteracting the tug of Moon and Sun on Earth's equatorial bulge, which both attempt to erect Earth's rotational axis. Acting like a spinning

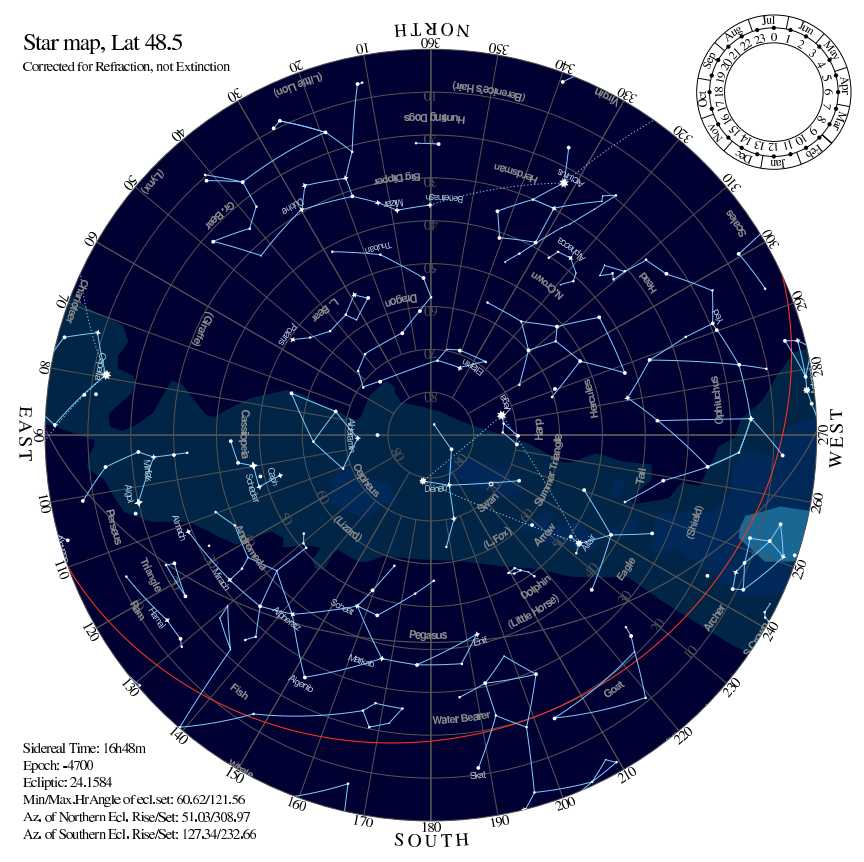

Figure 3: Typical all-sky map. When looking up from the Earth, cardinal directions appear mirrored compared to a geographical map. The red arc is part of the annual solar path, the ecliptic. The circle at upper right aligns mean solar time and calendar date to show when this map is applicable each day. The setting is for a situation in the year 4701 B.C. (astronomical -4700), described later in the text (Section 4).

top, Earth evades this erection by moving its axis sideways. This results in a motion where the Earth's axis over about 25800 years traces a double cone with the tip in the Earth's center. Reflecting this motion, the stars seem to move parallel to the ecliptic (the red line in figure 3), slowly changing their coordinates on the celestial sphere. For this reason, the star we now know as Pole Star will be far from the pole in just a few centuries, rendering it unusable for navigational purposes. The neolithic sky was shifted by about $90^{\circ}$ from today's sky.

Meeus [6] describes algorithms to correct stellar catalog positions given for one epoch to another.

If a structure's date cannot be determined to at least few hundred years, searching for alignments with stars is useless, because almost every azimuth will be covered by some bright star at some point in time. Here, only solar and lunar alignments can be investigated.

Fortunately, the epoch where Kreisgrabenanlagen were in use is known and is even short enough to use a single mean date for all sites without too great errors. The effect of precession on declination $\delta$ (and thus, combined with latitude $\varphi$, on rising and setting points) will be strongest where the ecliptic crosses the equator. In the latitude of $\varphi=48.5^{\circ}$, during the 300 year period, the azimuth shift amounts to about $1.7^{\circ}$. Lines inside the KGAs cannot be given to sub-degree accuracy, because the exact positions of, e.g., post tops above the surface is lost and can only be estimated from post hole positions.

\section{Mapping The SKY DOME}

For archeo-astronomical research, a method has to be found to combine archeological data, e.g., an excavation map, with astronomical data, i.e., positional data of celestial objects. Research typically 


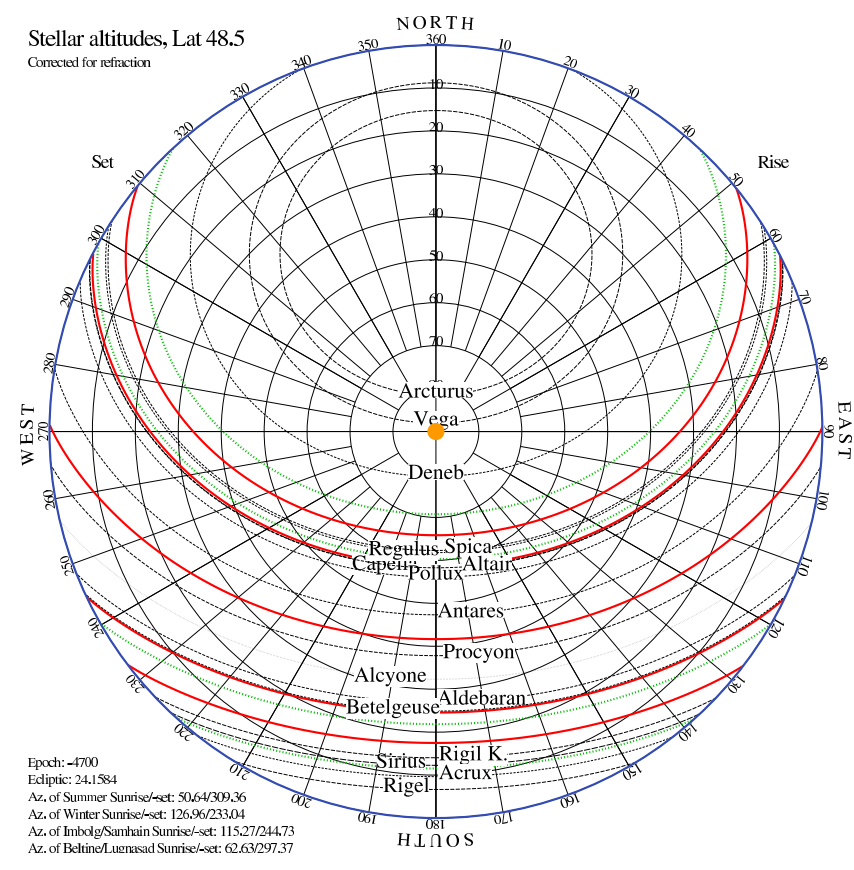

Figure 4: Map showing daily paths of brighter stars over the sky, together with the Sun's path on 8 specific dates of the year (red) and extreme paths of the Moon (green). The viewer is looking downward from the outside of the apparent sky dome, so the cardinal directions are aligned like on an ordinary geographical map. The paths change over centuries due to precession and (much less) stellar proper motion. Rising and setting directions can be read off at the horizon line (blue). Like in figure 3, the map is centered around the zenith (orange dot).

concentrates on alignments of artificial structures towards rising and setting points of Sun, Moon and selected bright stars.

Earlier studies frequently just pointed out orientations towards these directions on a map, assuming a flat horizon, because surveyed horizon data was rarely available and data for zero altitude are easy to compute. However, horizon profiles nowadays can be acquired from a Geographical Information System (GIS), so a diagram combining the archeological structure, sky dome and horizon had to be created.

\subsection{All-Sky Map}

A common way to show an all-sky star map, sometimes found in newspaper columns, consists of a fisheye-like round star map either in stereographic projection or with linearly mapped zenith distance (Figure 3). In the linear mapping, a point with azimuth $A$ and elevation $h>0$ on the hemisphere is mapped to a point with radius $r=90^{\circ}-h$ and angle $\theta=A$, counted counterclockwise from top. The zenith in the center shows stars immediately overhead at a certain time, whereas the stars close to the outer border are low on the horizon.

This mapping nicely shows an instantaneous view of the sky, but is almost totally useless for investigations of points on the horizon where the Sun or stars rise or set.

\subsection{Star Path Map}

Our interest while investigating astronomical alignments does at first not involve an instantaneous view, but a representation of all possible positions a star can reach for a certain geographical location. This leads to a map showing the daily paths of the stars

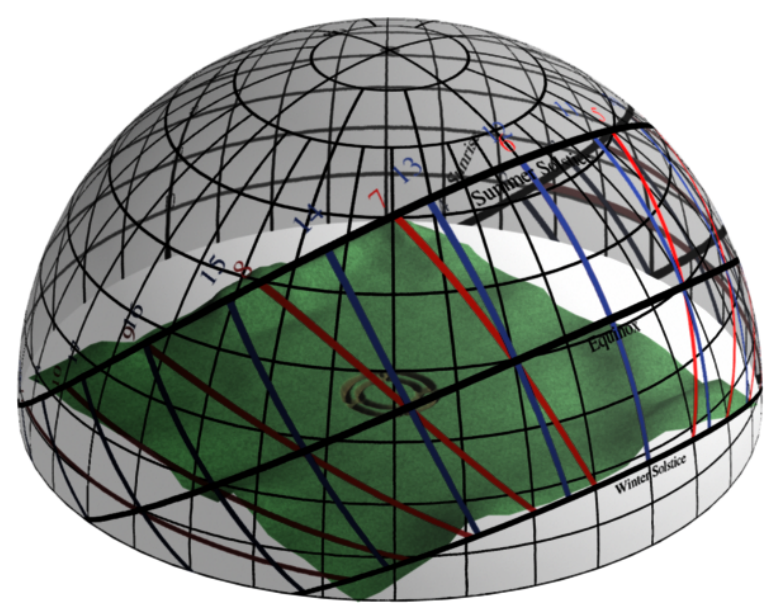

Figure 5: A mapping similar to Fig. 4 showing only the Sun's annual sky coverage used in a 3D scene, viewed from south-west. Mapped on an infinite sphere, a virtual walkthrough of an archaeological site will show possible alignments.

(Figure 4). In this figure, we exchanged east and west to provide a mapping which represents a view from "outside" the local sky dome, so east and west are oriented like on an ordinary geographical map, and $\theta=A$, but counted clockwise from top. This map allows to read the rising and setting points of celestial objects on the outer border, and also the altitudes and combined azimuths an object can reach.

\subsubsection{Application for a 3D Scene}

The map of figure 4 is perfectly usable as texture on a large (infinite, if possible) hemisphere for a virtual reconstruction inside a 3D modeling or VR application (Figure 5). During a virtual walkthrough, astronomical alignments can be identified. However, 3D reconstructions of archaeological sites including their surrounding landscapes require lots of effort, so a simpler way had to be found.

\subsection{Flipped Star Path Map}

Our aim was to find a mapping in which the orientation lines (ditches, palisade gaps) can be combined with points on the horizon which in nature lie outward of the structure. To achieve this, we must leave the traditional mapping of the hemisphere to the circle with the zenith in the center. Instead, we flip the mapping by ripping the sky dome open at the zenith and "folding" the sky outward. This creates a map with an opening in the center, where we leave room for other data, and the whole sky flattened outward, with the point of the zenith mapped to the outermost circle. So, $\theta=A$, counted clockwise from north like on a geographical map, but $r=r_{0}+h$, where $r_{0}$ is the radius of the inner area. The star paths appear largely unnatural at first but are still readable. Rising and setting points can be read again on the horizon, which is now the inner circle (Figure 6).

The red arcs in Figures 4 and 6 represent the Sun's paths at the dates of solstices (northern-/southernmost), equinoxes (east to west) and dates lying between these dates. Dashed green lines represent the standstill lines of the Moon. Star paths are marked in black arcs with various dot patterns according to the brightness of the respective star. The maps show star paths of the year 4701 B.C. (the astronomical year -4700 ).

This mapping now provides an inner area, which we used for two distinct purposes: Investigations of singular KGAs and an interpretation of a histogram of all identified directions. 


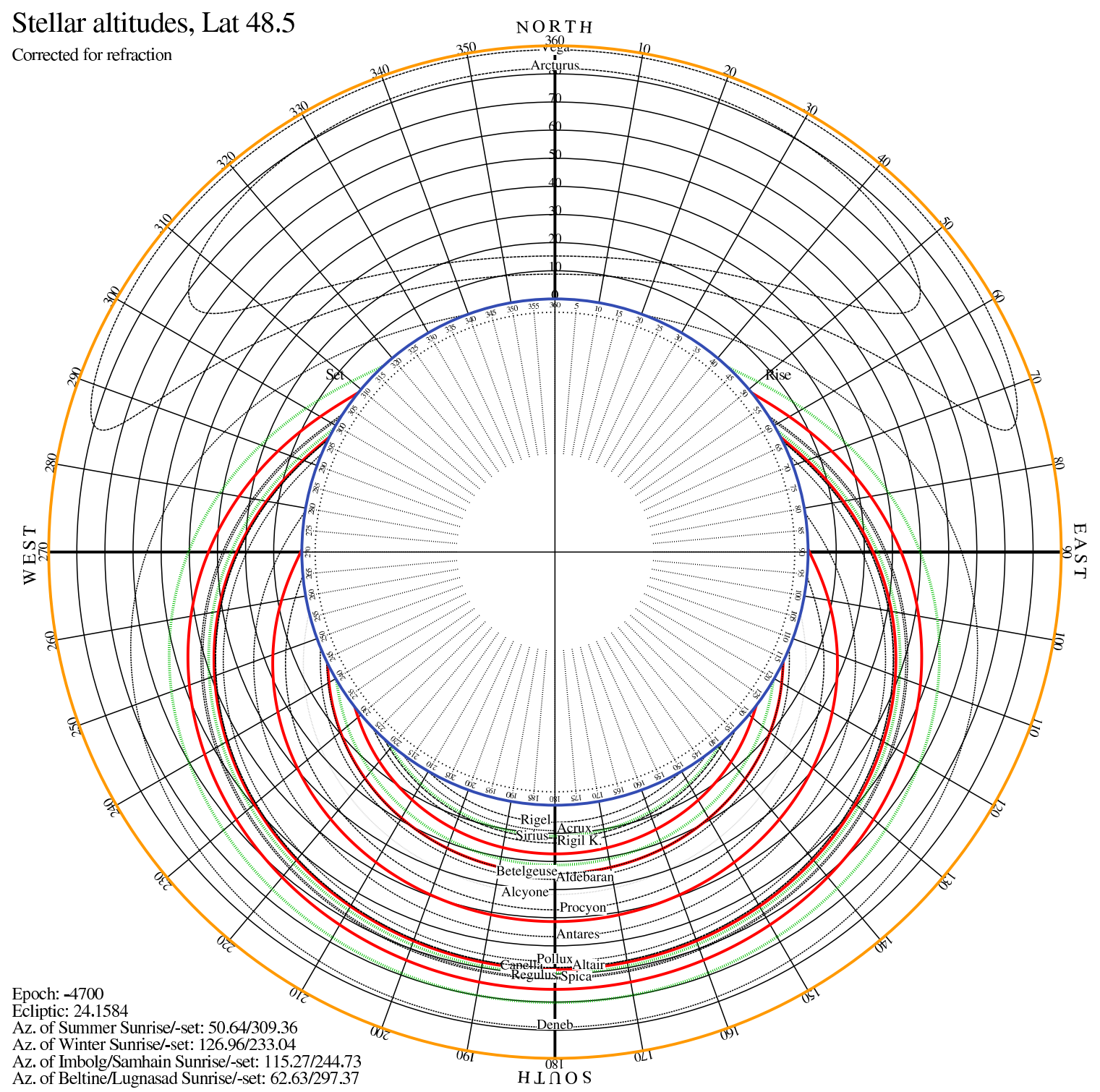

Figure 6: Flipped view of figure 4. The zenith (orange) is now mapped to the outermost circle, while the horizon (blue) encloses the inner area.

\subsubsection{Identification of Alignments}

When the center of the map is used as observer position and moved through the archaeological map, plausible alignments can be located. For a preliminary study, the diagram was printed on an overhead transparency and used with hardcopy prints of the archaeological artifacts.

For more thorough investigations concerning phenomena on the horizon (rising, setting), a local horizon must and can be added, slightly shifting all rising and setting events southward (on the northern hemisphere). We used horizon data extracted from $\mathrm{Ge}$ ographical Information System (GIS) data. Not all radial lines in KGAs intersect in a common center, so loading the KGA map and flipped star map into separate layers of a graphical editor allowed shifting of the KGA map and thus selecting the exact observer position, which was usually still close to the center (Figure 7). Nonradial directions have been marked with their respective orientations, allowing to read the intended orientation from the star map.

\subsubsection{Orientation Histogram}

All plausible orientation lines (views over earth bridges, along radial ditches or through palisade gaps from viewpoints close to the center) of 28 Kreisgrabenanlagen were entered in a circular histogram placed inside the mapping from figure 6. This histogram, in combination with the star path map lying immediately outward, allowed to identify groups of alignments which indicate these directions being chosen so that the objects rising or setting in these directions can be observed by looking e.g. along a ditch or through a door in the palisade (Figure 8).

Note that this histogram only works if the horizons are not highly elevated and if all sites approximately share geographical latitude. Due to the fact that an elevated horizon shifts both rising and setting points southward (on the northern hemisphere), the peaks in the histogram are a bit widened, but towards the south only. The widening is greater along the northern and southern parts of the horizon, caused by the shallow angles of the daily paths in these areas. 


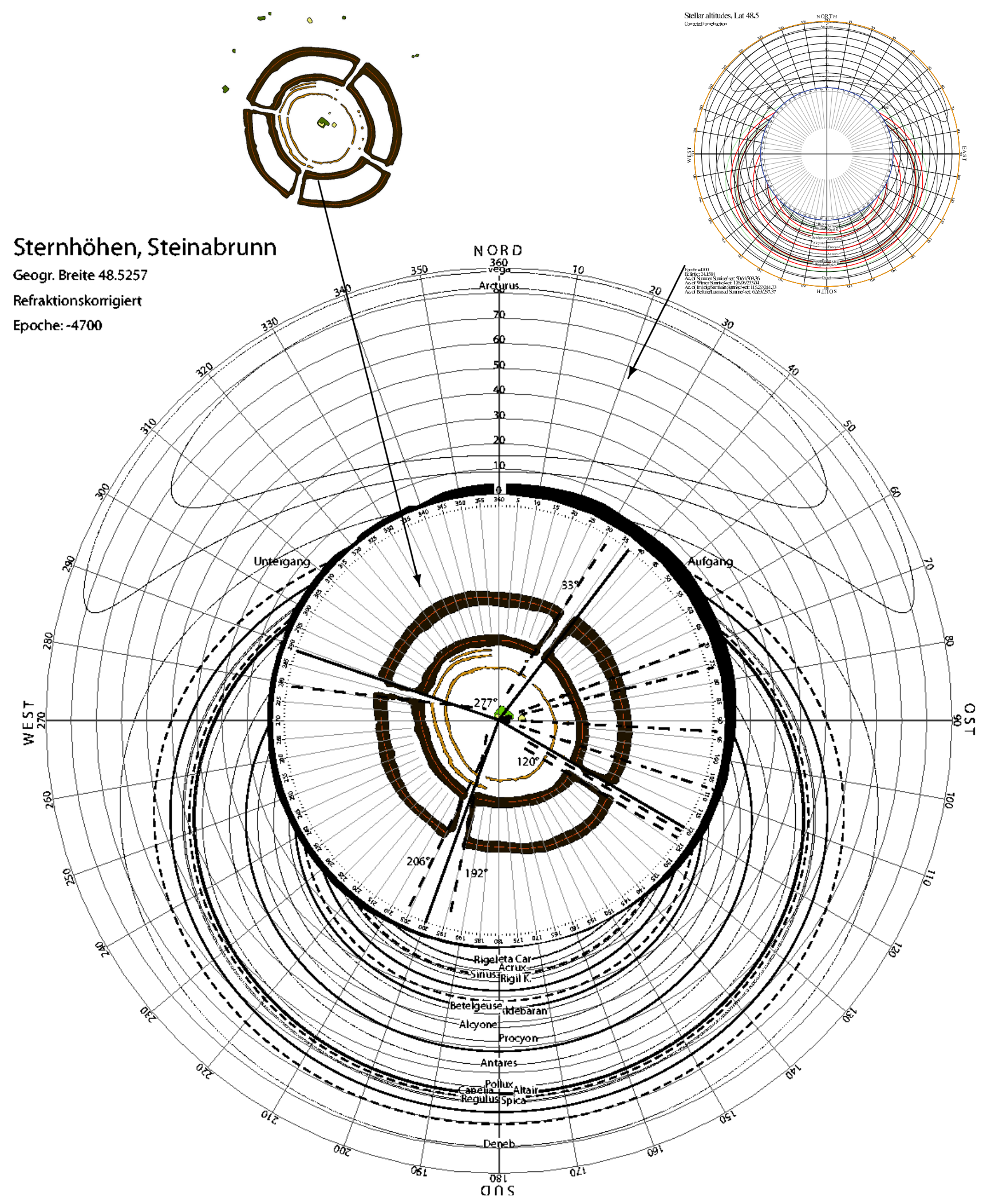

Figure 7: Combining the flipped star path map of figure 6 with data of the local horizon from GIS data (the thick irregular black zone along the horizon) and an archaeological map of the twofold Kreisgrabenanlage (KGA) of Steinabrunn [3] as example for an archaeological application. The radial lines indicate some of the plausible viewing directions along ditches, while the dashed lines indicate views through palisade gaps. Degree labels have been added where a line does not originate in the diagram's center. 

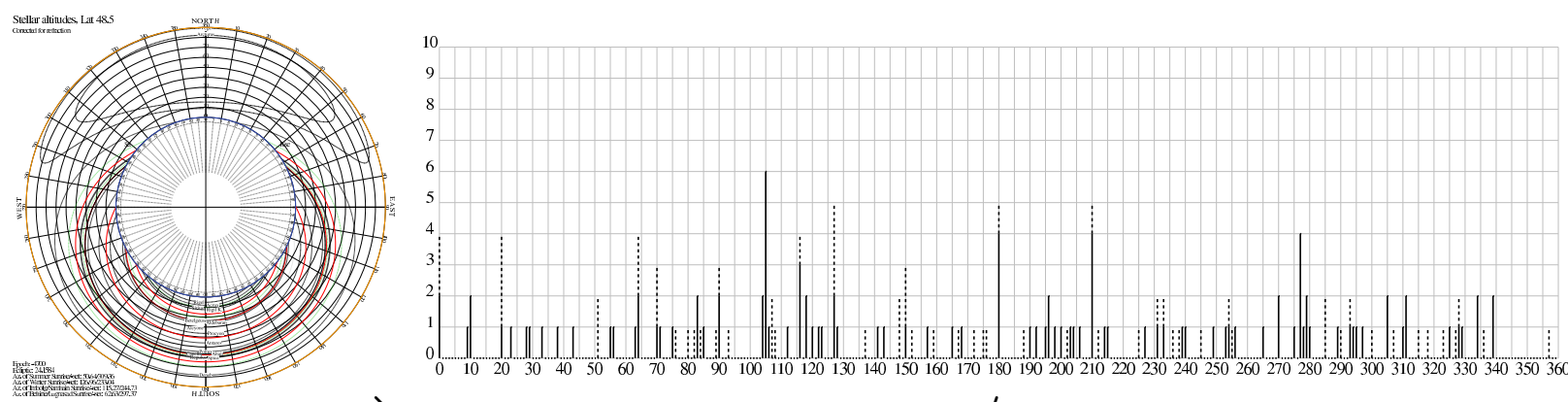

Histogram, Lat 48.5

Corrected for refraction

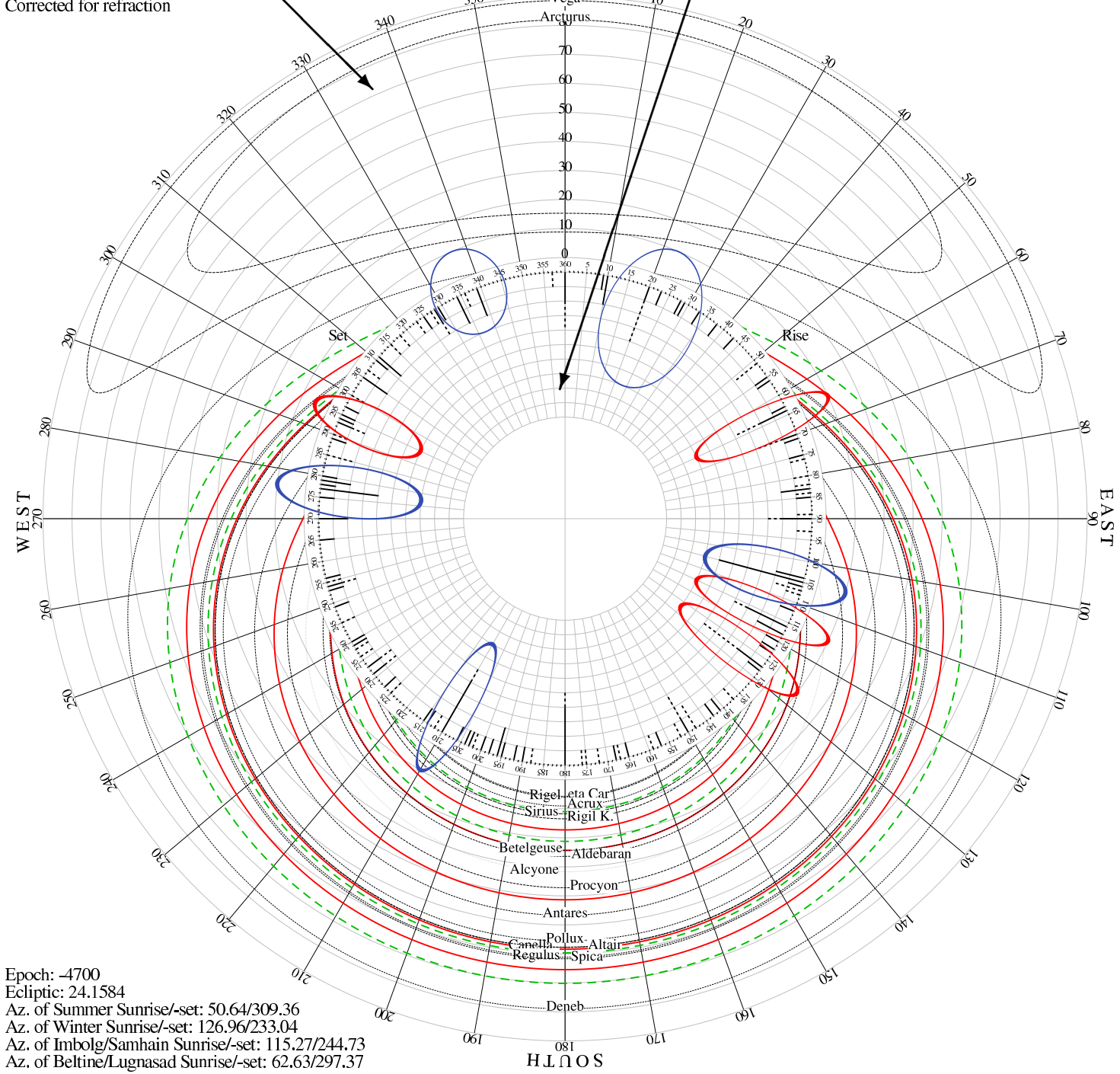

Figure 8: An ordinary rectangular histogram of all identified directions over azimuths of 28 Kreisgrabenanlagen (top right). The thick lines indicate the number of orientation lines pointing toward the respective azimuth. Full lines indicate directions of radial ditches or earth bridges, dashed lines indicate less conclusive gaps in the palisades. The histogram shows some peaks, but is hard to understand. Putting the histogram inside the mapping of figure 6 , with lines pointing from the horizon circle towards the center, immediately shows the possible astronomical connection of these directions. North and south are clearly indicated in 2-4 resp. 4-5 KGAs, east and west only in 2-3 each. A few peaks indicate alignments to certain solar dates (marked with red ellipses): directions around $127^{\circ}$ indicate Winter solstice sunrise, $115^{\circ}$ and slightly southward (shifted by raised local horizons at several sites) apparently mark sunrise at start and end dates of a "Winter" season centered around solstice. A weak group near $64^{\circ}$ and a larger group near $295^{\circ}$ may indicate sunrise and sunset at beginning or end of a "Summer" season similarly centered around summer solstice, which is not strongly marked itself (at $50^{\circ}$ ). The strongest peaks however appear to be connected to stars (blue ellipses). The strongest concentration lies in the range 104-108 ${ }^{\circ}$ with in total 9 strong and 3 more weak hits, which indicates the rising point of the Pleiades star cluster, marked here by the path of its brightest star, Alcyone. Almost on the opposite side, the setting direction of Antares is pointed to by 8 or even 9 strong hits in the range $275-280^{\circ}$. The calendarical interpretation of these peaks is described in section 4. 


\section{DisCOVERIES}

Combined with either interactive desktop planetarium software or a self-made planisphere for the neolithic period, a skilled astronomer can interpret a few of the detected accumulations of azimuth directions. In addition to the factual alignment visible from the diagram, we wanted to solve the question why certain stars could have been so important that our far ancestors oriented their largest buildings after them.

For the Sun, the most obvious direction accumulations are near azimuth $127^{\circ}$ (Winter solstice) and south of $115^{\circ}$ (Candlemas/AllSaints-Day), thus marking start, middle and end of a "Winter" season centered around Winter solstice. On the other side, Summer appears to be marked mostly with begin/end dates.

Still, it seems evident that not only solar directions have played a role in the orientations of Kreisgrabenanlagen. While the data currently do not offer a clear connection to lunar extremal risings or settings (dashed green lines in figures 4 and 8), there are two striking peaks towards directions $104-108^{\circ}$ and $275-280^{\circ}$. Indeed, around 4700 B.C., there have been two very conspicuous objects rising resp. setting at these directions: the Pleiades star cluster (rising) and the bright star Antares in Scorpius (setting). Moreover, the events of rising Pleiades and setting Antares took place almost simultaneously. Further investigation led to the conclusion that the Pleiades' heliacal rising (see section 2) took place just after spring equinox. It appears very likely that the neolithic farmers used this celestial event to start their agricultural year. Note that the Pleiades have been observed by many cultures worldwide in connection with the seasons [5], although a star cluster like the Pleiades, which consists of dim stars, cannot be observed below an extinction angle of about $a_{E}=4^{\circ}$, which is, however, a typical horizon elevation in Lower Austria.

Deneb, the Swan's tail star, joins the couple: just when Antares is setting and the Pleiades are rising, Deneb is highest in the sky, shining almost in the zenith. It is the northernmost bright star that sets below the northern horizon, and a wide group of doors and other sight lines appear to point towards it. Unfortunately, the intersections on the northern and southern horizon are very sensitive to elevated horizon lines, so the entries in the histogram, which cannot contain a horizon line, appear less concentrated. Figure 3 shows the scene where simultaneously Antares is setting near $275^{\circ}$, the Pleiades rise at $106^{\circ}$ and Deneb culminates in the south in about $80^{\circ}$ altitude (near center).

Also a frequently used southern gate direction $\left(210^{\circ}\right)$ required to look for a star setting there. Unfortunately, there are several stars sharing almost the same declination, but Rigel appears to be the brightest candidate. Further investigations in the field, taking measured horizon profiles, should clarify and narrow the error margins for this and all other directions.

\section{CONClusion And Future Work}

In this paper we have shown a simple, yet effective way to investigate buildings with known date of origin for astronomical orientations towards special rising and setting points of Sun, Moon and stars. The core item is a novel diagram combining a terrestrial map, e.g., of a building or other archaeological structure, and a foldedapart representation of the sky dome showing daily paths of celestial objects and the local horizon. We have shown how this diagram has been applied to a certain class of neolithic circular enclosures (Kreisgrabenanlagen), and have pointed out interesting discoveries made with a variant of the diagram which includes a histogram of alignments of a larger number of KGAs. These discoveries allowed us to produce renderings for selected points of view inside virtual reconstructions. These were used as foreground panoramas for commercial desktop planetarium software to create astronomi- cal vistas and animations (e.g., Figure 1 [12]). Clearly, an optimal solution would be an interactive, navigable VR/AR environment with an astronomically correct and visually convincing high quality sky simulation, which would allow immediate investigation of virtual reconstructions of archaeological sites. Unfortunately, only partial solutions of such systems seem to exist (e.g., [8]).

The concept of analyzing collected azimuth directions in a circular histogram could be extended to allow interactive work. Interactive brushing through a larger database of KGAs in a Geographical Information System (GIS) which includes their possible orientations and selecting, e.g., only archaeological sites in a specific region, could for example probably identify local traditions, although the total number of known KGAs in Europe (about 120 [2, p.4]) may be too small to deliver reliable results. If data from a long temporal series (several centuries) are investigated, the star paths will be seen shifting due to precession, still, for example an interactive investigation of the orientation of burial sites could probably find long-lasting traditions. In any case, dating the archaeological findings is necessary for any stellar position related investigation.

\section{ACKNOWLEDGeMENTS}

Data of Kreisgrabenanlagen and local horizons have been provided by Wolfgang Neubauer. The first author would like to thank Michael Gervautz and Heimo Kramer for the interesting discussion during development of the flipped star map, and Ralf Habel for Figure 5. Thanks also go to the anonymous reviewers.

This work was supported by the Austrian Science Fund (FWF) under contract number P17558.

\section{REFERENCES}

[1] Helmut Becker. Kultplätze, Sonnentempel und Kalenderbauten aus dem 5. Jahrtausend vor Chr. - Die mittelneolithischen Kreisanlagen in Niederbayern. Arbeitshefte des Bayerischen Landesamtes für Denkmalpflege, (59), 1996.

[2] Falko Daim and Wolfgang Neubauer, editors. Geheimnisvolle Kreisgräben - Niederösterreichische Landesausstellung 2005, Horn, Wien, 2005. Verlag Berger.

[3] Alois Eder-Hinterleitner, Wolfgang Neubauer, and Peter Melichar. Magnetic modelling for the 3D reconstruction of the neolithic circular ditch system of Steinabrunn/Austria. In J. Faßbinder and W. Irlinger, editors, Archaeological Prospection, number 108 in Arbeitshefte des Bayerischen Landesamtes für Denkmalpflege, pages 32-33. 1999.

[4] Maria G. Firneis. Untersuchung zur astronomischen Orientierung des Domes zu St. Stephan/Wien. Mitt. d. MathematischNaturwissenschaftlichen Klasse, Österreichische Akademie der Wissenschaften, 193(8-10), 1984.

[5] E. C. Krupp. Echoes of the Ancient Skies - The Astronomy of Lost Civilizations. Oxford University Press, New York, Oxford, 1994.

[6] Jean Meeus. Astronomical Algorithms. Willmann-Bell, Richmond, Virginia, second edition edition, 1998.

[7] Rolf Müller. Der Himmel über dem Menschen der Steinzeit, volume 106 of Verständliche Wissenschaft. Springer-Verlag, BerlinHeidelberg-New York, 1970.

[8] UCLA Visualization Portal. vrNav2. http://www.ats.ucla. edu/portal/research_activities/vrNav/default.htm, access 07/2005.

[9] Wolfhard Schlosser and Jan Cierny. Sterne und Steine - Eine praktische Astronomie der Vorzeit. Wissenschaftliche Buchgemeinschaft, Darmstadt, 1996.

[10] Alexander Thom. Megalithic Sites in Britain. Clarendon Press, Oxford, 1967.

[11] Alexander Thom and Alexander S. Thom. Megalithic Remains in Britain and Brittany. Clarendon Press, Oxford, 1978.

[12] Georg Zotti. Kalenderbauten?-Zur astronomischen Ausrichtung der Kreisgrabenanlagen in Niederösterreich. In Daim and Neubauer [2], pages $75-79$. 